\title{
Characteristics of and management strategies for 54 suicidal inpatients in a general hospital ${ }^{\dagger}$
}

Original article

Rong Tana, De-Ying Hü ${ }^{a}$, Yan-Hong Hanª, Yi-Lan Liua, Xiao-Ping Ding ${ }^{a}$, Shu-Jie Wang ${ }^{b}$, Ke Xua

aThe Union Hospital, Tongji Medical College, Huazhong University of Science and Technology, Wuhan, Hubei 430022, China

${ }^{b}$ Department of Nursing Science, Tongji Medical College, Huazhong University of Science and Technology, Wuhan, Hubei 430030, China

Received: 31 August 2017; Accepted: 5 October 2017; Published: 20 June 2018

\begin{abstract}
The aim of this study was to explore the characteristics of and preventive management strategies for suicidal inpatients in a general hospital.

Methods: $\mathrm{A}$ total of 54 suicide victims were drawn from a patient safety adverse event network reporting system during hospitalization in a general hospital from November 2008 to January 2017.

Results: Subjects who committed suicide in the general hospital were women and those who suffered from malignant neoplasms during general hospital treatment. Furthermore, most of the patients who committed suicide used more violent suicide methods. The most common and lethal means was jumping from heights at the windowsill.

Conclusions: It is concluded that management strategies for suicide prevention can be provided from the aspects of patients, medical staff and the hospital environment. It is not only urgent but also feasible to reduce the suicide rate of inpatients and further improve hospital safety management.
\end{abstract}

Keywords: inpatients $\bullet$ suicide $\bullet$ general hospital $\bullet$ management strategy $\bullet$ prevention

(c) Shanxi Medical Periodical Press.

\section{Introduction}

Suicide is a crucial global public health problem. According to the latest statistics, in 2012, there were approximately 803,900 people who committed suicide worldwide; approximately 120,730 of those suicide deaths occurred in China, where the suicide rate is 8.7 per 100,000 inhabitants and is estimated to account for $15 \%$ of suicides worldwide. ${ }^{1}$ The World Health Organization has predicted that by 2020 , mental disorders and suicide will become first on the list of total burden from diseases in China. ${ }^{2}$

Patient safety has increasingly become an important concern in modern healthcare systems, and inpatient ${ }^{+}$The project was supported by the National Natural Science Foundation
of China (No. 71673100).

* E-mail: hudeying2006@126.com suicide is the second most common alert event reported to the Joint Commission on Accreditation of Healthcare Organizations. ${ }^{3}$ It has been reported that the incidence of suicide is $5-15$ per 100,000 admissions in general hospitals, which is four to five times more than in the general population. ${ }^{4,5}$ Inpatient suicide is an adverse event that directly affects patient safety in the general hospital. Even in the medical setting, both medical staff and patients are confronted in courts due to some inpatients having committed suicide in the hospital. ${ }^{6}$ Obviously, inpatient suicide creates great distress among the patient's family members and has devastating effects on survivors and staff. It is of great theoretical and practical significance to study suicide in hospitalized patients, which can help reduce medical disputes, enhance the doctor-patient relationship and improve hospital reputation. 
The aims of this study were to examine the patient safety adverse event network reporting system among suicide victims in Chinese hospitals and to analyze suicidal characteristics and relevant causes. Furthermore, it is not known if attempted suicidal inpatients differ from subjects who succeed in killing themselves. We also aimed to compare the suicide characteristics of these two populations and ultimately provide preventive nursing management strategies.

\section{Material and methods}

\subsection{Study sample}

This retrospective study was conducted in a large general hospital with 5,000 beds that provides treatment for more than 200,000 inpatients per year. The data were based on a patient safety adverse event network reporting system in which suicidal cases occurred in one hospital from November 2008 to January 2017. Information included patients' demographic data (age, gender, marriage, date of admission, medical diagnosis, department, etc.), details of the event (time, methods and location of occurrence, etc.), reason or condition leading to the suicidal act, outcome and suggested methods to prevent recurrence.

\subsection{Statistical analysis}

Statistical analyses were performed with Microsoft Excel spreadsheets and SPSS (version 17.0; SPSS Inc., Chicago, IL, USA). Mean, standard deviation and percentage were used for descriptive analysis. The $t$-test was used to compare the different suicide methods by gender, and the chi-squared test was used to compare characteristics between patients who attempted suicide and patients who completed suicide. In statistical testing, $P<0.05$ was considered as significant.

\section{Results}

\subsection{Demographic characteristics}

A total of 54 cases were identified during the 9-year period. There were 29 women $(53.70 \%)$ and 25 men $(46.30 \%)$, with a female:male ratio of $1.16: 1.0$. The age of cases ranged from 20 to 80 years, and the mean age of cases was $54.87 \pm 12.38$ years. The mean age was $54.60 \pm 11.63$ years and $55.10 \pm 13.20$ years, respectively, for men and women. Almost all cases (92.59\%) were married. Females were more common among those who attempted suicide than among those who completed suicide; moreover, there was a significant difference for gender with regard to attempted and completed suicidal inpatients $\left(\chi^{2}=4.562, \quad P=0.033\right)$. Most cases were in the $45-59$ years age group $(n=25$, $46.30 \%$ ), followed by those above the age of 60 years $(n=18,33.33 \%)$, but no significant difference in age between attempted and completed suicidal inpatients was found $\left(\chi^{2}=2.216, P=0.330\right.$ ) (Table 1$)$, which may be related to the insufficient sample size of this study.

\subsection{Medical diagnoses and departments}

Among the 54 cases, 22 (40.74\%) patients had been treated in medical departments, $16(29.63 \%)$ in surgical departments, $14(25.93 \%)$ in the oncology center and two $(3.70 \%)$ in the obstetrics and gynecology department. The most common medical diagnosis among suicidal inpatients was malignant neoplasms $(n=35$, $64.81 \%)$, followed by digestive diseases $(n=5,9.26 \%)$, cardiovascular diseases $(n=5,9.26 \%)$ and other diseases $(n=9,16.67 \%)$. Medical diagnoses among patients who had attempted or completed suicide were not significantly different $\left(\chi^{2}=5.811, P=0.121\right)$ (Table 1$)$. Among the malignant neoplasms, gastrointestinal cancer and leukemia or lymphoma accounted for $14.81 \%$ of cases, followed by lung cancer $(n=7,12.96 \%)$, cervical carcinoma $(n=3,5.56 \%)$, pancreatic cancer $(n=3$, $5.56 \%)$, liver cancer $(n=3,5.56 \%)$ and other cancers $(n=3,5.56 \%$ ). Medical diagnoses of suicidal inpatients did not significantly differ by gender $(t=0.03, P=0.722)$ (Table 2).

\subsection{Length of stay before suicide}

Length of stay before suicidal behavior covered a wide range (1-42 days) with a mean of $11.31 \pm 9.60$ days. Completed suicides occurred at a mean of $11.33 \pm 9.03$ days after admission, and attempted suicides occurred at a mean of $11.33 \pm 10.18$ days after admission. Male and female inpatients did not have a significantly longer length of stay from admission to suicidal behavior $(t=0.474, P=0.801)$. Suicidal acts were most likely to occur in the first $(n=25,46.30 \%)$ and second $(n=11$, $20.37 \%$ ) weeks of admission. Among the 24 suicide completers, $11(45.83 \%)$ completed within the first week and four $(16.67 \%)$ completed in the second week (Table 1$)$.

\subsection{Time of suicidal act}

Both attempted and completed suicides had peak hours, which occurred between 2 a.m. and 6 a.m. Among the 54 patients, $16.67 \%$ of suicides occurred between 6 a.m. and 10 a.m., $3.70 \%$ between 10 a.m. and 2 p.m., $18.52 \%$ between 2 p.m. and 6 p.m., $16.67 \%$ between 
R. Tan et al.

\begin{tabular}{|c|c|c|c|c|}
\hline Items & $\begin{array}{l}\text { Attempted suicide } \\
\qquad(n=30)\end{array}$ & $\begin{array}{l}\text { Completed suicide } \\
\qquad(n=24)\end{array}$ & Total $(n=54)$ & Statistics \\
\hline \multicolumn{5}{|l|}{ Gender } \\
\hline Male & $10(33.33)$ & $15(62.50)$ & $25(46.30)$ & \multirow{2}{*}{$P=0.033^{*}$} \\
\hline Female & $20(66.67)$ & $9(37.50)$ & $29(53.70)$ & \\
\hline \multicolumn{5}{|l|}{ Age (years) } \\
\hline $18-44$ & $8(26.67)$ & $3(12.50)$ & $11(20.37)$ & \multirow{3}{*}{$P=0.330$} \\
\hline $45-59$ & $14(46.67)$ & $11(45.83)$ & $25(46.30)$ & \\
\hline$\geq 60$ & $8(26.67)$ & $10(41.67)$ & $18(33.33)$ & \\
\hline \multicolumn{5}{|l|}{ Medical diagnoses } \\
\hline Malignant neoplasms & $18(60.00)$ & 17 (58.33) & $35(64.81)$ & \multirow{4}{*}{$P=0.121$} \\
\hline Digestive diseases & $1(3.33)$ & $4(16.67)$ & $5(9.26)$ & \\
\hline Cardiovascular diseases & $4(13.33)$ & $1(4.17)$ & $5(9.26)$ & \\
\hline Other diseases & 7 (23.33) & $2(8.33)$ & $9(16.67)$ & \\
\hline \multicolumn{5}{|l|}{ Department } \\
\hline Medical department & $8(26.67)$ & $14(58.33)$ & $22(40.74)$ & \multirow{4}{*}{$P=0.092$} \\
\hline Surgical department & $11(36.67)$ & $5(20.83)$ & $16(29.63)$ & \\
\hline Oncology center & $9(30.00)$ & $5(20.83)$ & $14(25.93)$ & \\
\hline Obstetrics and Gynecology & $2(6.67)$ & 0 & $2(3.70)$ & \\
\hline \multicolumn{5}{|c|}{ Length of stay before suicide (days) } \\
\hline$\leq 7$ & $14(46.67)$ & $11(45.83)$ & $25(46.30)$ & \multirow{5}{*}{$P=0.251$} \\
\hline $8-14$ & 7 (23.33) & $4(16.67)$ & $11(20.37)$ & \\
\hline $15-21$ & $5(16.67)$ & $5(20.83)$ & $10(18.52)$ & \\
\hline $22-28$ & $1(3.33)$ & $4(16.67)$ & $5(9.26)$ & \\
\hline$\geq 29$ & $3(10.00)$ & 0 & $3(5.56)$ & \\
\hline \multicolumn{5}{|l|}{ Time } \\
\hline $2: 00-6: 00$ & 10 (33.33) & $10(41.67)$ & $20(37.04)$ & \multirow{6}{*}{$P=0.608$} \\
\hline $6: 00-10: 00$ & $5(16.67)$ & $4(16.67)$ & $9(16.67)$ & \\
\hline 10:00-14:00 & $1(3.33)$ & $1(4.17)$ & $2(3.70)$ & \\
\hline $14: 00-18: 00$ & $5(16.67)$ & $5(20.83)$ & $10(18.52)$ & \\
\hline 18:00-22:00 & $5(16.67)$ & $4(16.67)$ & $9(16.67)$ & \\
\hline $22: 00-2: 00$ & $4(13.33)$ & 0 & $4(7.41)$ & \\
\hline \multicolumn{5}{|l|}{ Methods } \\
\hline Jumping from heights & $8(26.67)$ & $17(70.83)$ & $25(46.30)$ & \multirow{6}{*}{$P=0.005^{*}$} \\
\hline Cutting & $13(43.33)$ & $2(8.33)$ & $15(27.78)$ & \\
\hline Drug overdose & $3(10.00)$ & $1(4.17)$ & $4(7.41)$ & \\
\hline Hanging & $1(3.33)$ & $3(12.50)$ & $4(7.41)$ & \\
\hline Drowning & $1(3.33)$ & $1(4.17)$ & $2(3.70)$ & \\
\hline Others & $4(13.33)$ & 0 & $4(7.41)$ & \\
\hline \multicolumn{5}{|l|}{ Places } \\
\hline Windowsill & $8(26.67)$ & $17(70.83)$ & $25(46.30)$ & \multirow{4}{*}{$P=0.003^{*}$} \\
\hline Bedside & 16 (53.33) & $3(12.50)$ & 19 (35.19) & \\
\hline Toilet & $2(6.67)$ & $3(12.50)$ & $5(9.26)$ & \\
\hline Outside hospital & $4(13.33)$ & $1(4.17)$ & $5(9.26)$ & \\
\hline
\end{tabular}

Table 1. Characteristics of suicidal inpatients, $n(\%)$.

Note: ${ }^{*} P<0.05$ indicates a statistically significant comparison between attempted and completed suicidal inpatients. 


\begin{tabular}{lccc}
\hline Items & Male & Female & Total, $n(\%)$ \\
\hline \hline Malignant neoplasms & 14 & 21 & $35(64.81)$ \\
Gastrointestinal cancer & 2 & 6 & $8(14.81)$ \\
Leukemia or lymphoma & 4 & 4 & $8(14.81)$ \\
Lung cancer & 3 & 4 & $7(12.16)$ \\
Cervical carcinoma & 0 & 3 & $3(5.56)$ \\
Pancreatic cancer & 1 & 2 & $3(5.56)$ \\
Liver cancers & 3 & 0 & $3(5.56)$ \\
Other cancers & 1 & 2 & $3(5.56)$ \\
Digestive diseases & 3 & 2 & $5(9.26)$ \\
Chronic liver disease & 2 & 2 & $4(7.41)$ \\
Hemorrhage of digestive tract & 1 & 0 & $1(1.85)$ \\
Cardiovascular diseases & 3 & 2 & $5(9.26)$ \\
Other diseases & 4 & 5 & $9(16.67)$ \\
\hline
\end{tabular}

Table 2. Disease categories of suicidal inpatients.

6 p.m. and 10 p.m. and $7.41 \%$ between 10 p.m. and midnight. However, we did not find a significant effect of time on the occurrence of inpatient suicide $\left(\chi^{2}=3.600\right.$, $P=0.608$ ) (Table 1).

\subsection{Suicidal methods and locations}

Jumping from heights $(n=25,46.30 \%)$ and cutting $(n=15$, $27.77 \%$ ) were the most common methods of suicide, followed by drug overdose $(n=4,7.41 \%)$, drowning $(n=2$, $3.70 \%)$ and hanging $(n=4,7.41 \%)$. A total of $68 \%(17 / 25)$ of inpatients died as a result of jumping from heights, which is the most lethal method of suicide. Nearly twofifths of suicide attempters tried to harm themselves by using tools (scissors, fruit knives, etc.). One-third of suicide attempters used fatal methods (jumping from heights or hanging) that were similar to those used by suicide completers. There were 51 suicidal acts that occurred within the hospital, 25 (46.30\%) occurred from the windowsill and five (9.26\%) at the bedside and in the bathroom. Additionally, five suicides (13.3\%) occurred outside the hospital. The majority of attempted suicides occurred in inpatients' beds or inside the ward, and far more completed cases occurred from the windowsill. Evidences of significant differences were observed in the methods and locations of suicidal inpatients $\left(\chi^{2}=16.848, P=0.005\right.$ and $\chi^{2}=13.636, P=0.003$, respectively) (Table 1).

\subsection{Relevant causes of suicide}

As shown in Table 3, somatic disease accounted for $27.78 \%$ of the suicide acts. The second reason for

\begin{tabular}{lccc}
\hline Items & Male & Female & Total, $n(\%)$ \\
\hline \hline Somatic disease & 5 & 10 & $15(27.78)$ \\
Economic pressure & 7 & 6 & $13(24.07)$ \\
Hopelessness & 4 & 5 & $9(16.67)$ \\
Social support & 6 & 2 & $8(14.81)$ \\
Depression & 3 & 2 & $5(9.26)$ \\
Personality defect & 1 & 3 & $4(7.41)$ \\
\hline
\end{tabular}

Table 3. Relevant causes associated with suicide.

suicidal behavior was economic pressure (24.07\%). Hopelessness and poor social support were reported as $16.67 \%$ and $14.81 \%$, respectively, of the causes of suicide. Depression and personality defects accounted for $9.26 \%$ and $7.41 \%$, respectively, of the cases. Moreover, there was more than one relevant cause for each case.

\section{Discussion}

The results of this study show that most suicide cases were middle-aged women. The most frequently used method for suicide was jumping from heights. Most cases committed suicide from the windowsill of the hospital building and at midnight. Economic pressure, somatic disease, hopelessness, poor social support, depression and personality defects were all relevant causes for suicide behaviors.

\subsection{Analysis of the characteristics of suicidal inpatients}

In the present study, most suicide cases were women, which is consistent with a previous study. ${ }^{7}$ Compared with Chinese men, Chinese women are more sensitive and fragile and more likely to commit suicide when they encounter adverse stimuli. It is noteworthy as reported by Wint and Akil and Bostwick and Rackley that more men commit suicide than women in general hospitals ${ }^{8,9}$ and more women than men are initially more willing to seek help after experiencing frustration in a Western country.

Patients with malignant tumors had the highest rate of suicidal acts, which is consistent with the related literature..$^{10,11}$ Hung et al. showed that somatic disease, particularly malignant neoplasms, was the most common disease and increased the risk of suicide in hospitalized patients. ${ }^{12}$ These cancer patients all had suffered from despair, fear, severe pain, dysfunction or cognitive function, which directly affected their quality of life. Underlying these circumstances, depression and anxiety are expected, and suicidal ideation might appear among terminal patients. 
It is important to note that 25 subjects committed suicidal acts within the first week and 11 in the second week of admission, which is similar to the findings of Hung et al. ${ }^{12}$ These results may suggest that patients are more likely to carry out suicidal acts within the first week after admission. The time of suicidal act is important for suicide prevention. In our study, we found that $37.04 \%$ of acts occurred between 2 a.m. and 6 a.m. The result is similar to other reports. ${ }^{11,13}$ This fact might be attributed to patients' suicidal acts occurring in the early hours of the morning when supervision was sparser and families were absent; thus, there was a higher likelihood of success during this time period.

The most common and lethal means of suicide in general hospitals was jumping (25 cases). This result is similar to the findings of local Chinese articles. ${ }^{14-16}$ However, in Western populations, cutting is the most frequent method of suicide in general hospitals. ${ }^{17}$ The difference in suicidal methods appears to be culturally based. The choice of a suicide method is closely related to the availability of suicide tools. At present, the modern ward building of the general hospital is mostly high-rise; most of the hospital's balconies and windows are not closed or are semi-closed like the psychiatric hospital. Undoubtedly, it is convenient for the patient to commit suicide. Moreover, in this study, cutting was another frequent method of suicide (15 of 54 subjects) because of its easy access for hurting oneself in the ward.

It is reported that hopelessness might make suicide risk increase at least three times; moreover, sustained hopelessness is more predictive of suicidal behavior than occasional hopelessness. ${ }^{18}$ Patients suffering from depression might be a warning sign before suicidal acts, but unfortunately, depression is often neglected by the medical staff. ${ }^{19}$ Therefore, a comprehensive evaluation of suicide risk is critical in these patients. According to their suicide risk factors, more attention should be paid to patients' psychological state. Additionally, it is essential to develop and implement a targeted suicide prevention program for mental disorder patients. ${ }^{20,21}$

\subsection{Management strategies of suicidal inpatients}

There is no solution to avoid the suicidal potential of inpatients. Modifying the process of patient assessment and care, strengthening staff training and communication and improving the physical environment are critically important. Therefore, to reduce inpatient suicidal behavior and ensure inpatient safety, this paper explores suicide preventive management strategies from the aspects of patients, medical staff and hospital environment, and recommendations are provided for each of these domains.

\subsubsection{The aspect of patients}

\subsubsection{Identify the characteristics of high-risk suicidal patients}

According to the literature, high-risk suicidal patients have certain characteristics, so it is important to implement treatment plans and provide personalized care based on the patient's condition. The suicidal patients with a high risk of the following factors are included: (1) severe somatic disease or terminal cancer: helping patients understand basic information about the disease, reducing fear and anxiety about the disease, relieving pain and sleep disorders and improving the patient's ability to cope with the disease; (2) hopelessness: paying attention to patients' emotional changes and detecting psychological problems in time, creating individualized programs to help patients relieve negative emotions and finally, reconstructing the correct model of emotional management; (3) depression: focusing on early identification of patients with depression; when the general psychological intervention is ineffective, active referral treatment is essential for patients with severe depression so that these patients can be effectively treated; (4) suicidal history: paying attention to psychological and mental assessments; furthermore, more attention should be focused on the collection of patients or relatives with or without suicide histories, especially in admission assessments and (5) personality defects: encouraging patients to take an optimistic attitude to problems, guiding patients with positive resources against negative emotions and establishing a positive attribution style.

\subsubsection{Establish a psychological assessment mechanism of clinical patients}

Patient assessment is a continuous dynamic process. ${ }^{22}$ Making an initial assessment is the most important. Nurses should perform the overall assessment 2 hours after admission, according to the nursing process, and clear the patient's care needs. Medical staff can apply the mature psychological measurement scales, such as the Patient Health Questionnaire-9, ${ }^{23}$ Nurses' Global Assessment of Suicide Risk ${ }^{24}$ and SAD PERSONS scale,${ }^{25}$ to assess whether the patient is in a state of depression or anxiety. Second, the nurse is responsible for a reassessment and recording of the patient once a day, especially for some special patients (with malignancies, special examinations, surgery, condition or mood changes, etc.). Past suicide history, negative emotions and negative life events are key points of assessment. 
Finally, dynamic, continuous assessment is very necessary; nurses dynamically assess, analyze, master and monitor the psychological states of suicidal patients at a high risk and then start suicide contingency plans in order to reduce the risk of occurrence.

\subsubsection{The aspect of medical staff}

It is clear that medical staff undertake the role of gatekeeper in maintaining patient safety. There is a close connection between the mastery of suicidal knowledge, cognition of suicidal patients and patient safety. ${ }^{26} \mathrm{~A}$ previous study has shown that the root cause of suicide in hospital patients is the lack of training on the patient's psychological problems and coping ability. ${ }^{27}$ It is meaningful to prevent the suicide of hospitalized patients by training medical staff about suicide safety management. The first step is to carry out suicide knowledge training for nurses with lectures and continue education programs. The training content includes basic suicide knowledge, the identification of high-risk suicidal patients, suicide prevention measures and so on. The second step is skills training, teaching nurses to accurately and skillfully use various psychological scales, practicing and examining the suicide contingency plans to improve the emergency response ability of nurses and organizing nurses to learn how to communicate effectively with suicidal patients. The last step is attitude training; humanistic care should be integrated into the daily care of suicidal patients, and nurses' positive attitudes toward suicide should also be established so that the patients with suicidal tendencies can be intervened in time. Finally, it is necessary to conduct a specific quantitative examination of nurses' abilities and then to improve the theoretical level of nurses' knowledge and abilities to address suicide.

\subsubsection{The aspect of hospital environment}

A recent study highlighted that the hospital environment was the most common cause of suicide. ${ }^{28}$ That study indicated that emphasizing the physical environment of the hospital was an important strategy to reduce the overall risk of suicide and ensure patient safety. Mohl et al.

\section{References}

1. World Health Organization. Preventing Suicide - A Global Imperative. Geneva: WHO; 2014.

2. World Health Organization. The Global Burden of Disease. http://www.who.int/topics/global_burden of_disease/zh/.Accessed April 17, 2017 (in Chinese). have also shown that there is a significant reduction in suicide jumps following the installation of a metal guard rail at each window of the high-rise building. ${ }^{29}$ As a result, restricting access to lethal means is momentous. We can take the following measures: if possible, formulate management rules for doors, windows and knives and revise them yearly. Such measures might include setting up a special registration book with special personnel to inspect and record the condition of windows and knives every month; timely maintenance of the ward windows, corridors and balconies if they are damaged; fully refurbishing windows to prevent jumps from them, especially if someone jumped from a height in the ward and informing patients that knives are not allowed in the ward when they are admitted to the hospital and that nursing stations will reserve a standby blunt fruit knife if the patients need to cut food.

\section{Conclusions}

This retrospective study demonstrated the characteristics of suicidal acts occurring in general hospitals in the Hubei province. The patterns of regulation to cope with inpatient suicide should be improved. Appropriate management measures for suicidal patients at general hospitals from the aspects of patients, medical staff and hospital environment seem to be important. The ultimate aim is to provide references for clinical nursing managers to prevent and reduce suicidal behavior.

There are several limitations in this study: (1) since the inpatient sample was drawn from a single hospital, generalizability is limited and (2) the reporting system of this retrospective study had problems with information integrity, nonstandardized recording procedures and the probability of underestimating the true frequency of suicidal behavior. Our continuing efforts will extend this project to different grades of the hospital to collect factual data by using an improved reporting system. There is no doubt that it is more important to encourage nurses to report suicidal acts than to punish staff.

\section{Conflict of interest}

All contributing authors declare no conflicts of interest.
3. Cheng IC, Hu FC, Tseng MC. Inpatient suicide in a general hospital. Gen Hosp Psychiatry. 2009;31:110-115.

4. Shapiro S, Waltzer H. Successful suicides and serious attempts in a general hospital over 15-year period. Gen Hosp Psychiatry. 1980;2:118-126. 
5. Martelli C, Awad H, Hardy P. In-patients suicide: Epidemiology and prevention. Encephale. 2010;36 (suppl 2):D83-D91 (in French).

6. Phoenix Information. Patient suicide death in hospital, doctors argue with family members in the courtroom; 2017. http://news.ifeng.com/gundong/ detail_2014_03/17/34849864_0.shtml. Accessed March 17, 2017.

7. Liang $T$, Zhang $X Y$, Wang Z. A review on the suicide gatekeeper training. Adv Psych Sci. 2012;20: 1287-1295 (in Chinese).

8. Wint DP, Akil M. Suicidality in the general hospitalized patient. Hosp Physician. 2006;42:13-18.

9. Bostwick JM, Rackley SJ. Completed suicide in medical/surgical patients: Who is at risk? Curr Psychiatry Rep. 2007;9:242-246.

10. Liu $Q, X u Y$, Sun $Y L$. Death causes analysis of 1545 cases in general hospital. China $J$ Mod Med. 2008;18:2690-2692, 2695 (in Chinese).

11. Gong XM, Zhao JL, Zhu Y, Liu X. Analysis of suicide behavior of inpatients and nursing countermeasures. Mod Clinical Nurs. 2008;7:64-65, 72 (in Chinese).

12. Hung $\mathrm{Cl}$, Liu CY, Liao MN, Chang YH, Yang YY, Yeh EK. Self-destructive acts occurring during medical general hospitalization. Gen Hosp Psychiatry. 2000;22:115-121.

13. Lu CY, Xie HZ. Analysis on 19 suicide cases of inpatient in certain hospital. Hosp Admin J Chin PLA. 2014;21:488-490 (in Chinese).

14. Chen J. Analysis of suicidal behaviors of the inpatients in general hospitals and the psychological interventions. Nurs J Chin PLA. 2008;25:76-77 (in Chinese).

15. Liu H, Yan J. An analysis of the causes of suicide committing by jumping off buildings by 7 patients with physical diseases and the coping strategy. J Nurs. 2009;16:4-6 (in Chinese).

16. Suominen K, Isometsä E, Heilä H, Lönnqvist J, Henriksson M. General hospital suicides - a psychological autopsy study in Finland. Gen Hosp Psychiatry. 2002;24:412-416.

17. Carroll R, Thomas KH, Bramley K, et al. Self-cutting and risk of subsequent suicide. J Affect Disord. 2016;192:8-10.

18. McMillan D, Gilbody S, Beresford E, Neilly L. Can we predict suicide and non-fatal self-harm with the Beck Hopelessness Scale? A meta-analysis. Psychol Med. 2007;37:769-778.

19. Niu YJ, Wang ZQ, Yang SJ, Fei LP, Wang $X Q$. Survey of suicidal ideation and suicide attempts in inpatients at 40 general hospitals in Beijing. Chin Mental Health J. 2006;20:457-461 (in Chinese).

20. Al-Habeeb AA, Sherra KS, Al-Sharqi AM, Qureshi NA. Assessment of suicidal and self-injurious behaviours among patients with depression. East Mediterr Health J. 2013;19:248-254.

21. Jiang C, Li X, Phillips MR, Xu Y. Matched casecontrol study of medically serious attempted suicides in rural China. Shanghai Arch Psychiatry. 2013;25:22-31.

22. Joint Commission International. Joint Commission International Accreditation Standards for Hospital. 5th ed. Oakbrook Terrace, USA: Joint Commission International; 2014.

23. Spitzer RL, Kroenke K, Williams JB. Validation and utility of a self-report version of PRIME-MD: The $P H Q$ primary care study. Primary Care Evaluation of Mental Disorders. Patient Health Questionnaire. JAMA. 1999;282:1737-1744.

24. Cutcliffe JR, Barker P. The Nurses' Global Assessment of Suicide Risk (NGASR): Developing a tool for clinical practice. J Psychiatr Ment Health Nurs. 2004;11:393-400.

25. Patterson WM, Dohn HH, Bird J, Patterson GA. Evaluation of suicidal patients: The SAD PERSONS Scale. Psychosomatics. 1983;24:343345, 348-349.

26. Saunders KE, Hawton K, Fortune S, Farrell S. Attitudes and knowledge of clinical staff regarding people who self-harm: A systematic review. J Affect Disord. 2012;139:205-216.

27. Zhu XQ, Lu LJ, Yu Y, Hu DY. Analysis and thinking of root causes of suicide inpatients. Chin Nurs Res. 2015;29:3153-3156 (in Chinese).

28. Lieberman DZ, Resnik HL, Holder-Perkins V. Environmental risk factors in hospital suicide. Suicide Life Threat Behav. 2004;34:448-453.

29. Mohl A, Stulz N, Martin A, et al. The "Suicide Guard Rail": a minimal structural intervention in hospitals reduces suicide jumps. BMC Res Notes. 2012;5:408-408.

How to cite this article: Tan R, Hu DY, Han YH, et al. Characteristics of and management strategies for 54 suicidal inpatients in a general hospital. Front Nurs. 2018; 2: 139-146. https://doi.org/10.2478/fon-2018-0018 